\title{
SINTASSI, SEMANTICA, PRAGMATICA IN ALCUNI INTERESSANTI CASI DI AMBIGUITA NEI TESTI ISTROROMANZI MODERNI
}

1. Nell'ambito del nostro progetto di studio sintattico, semantico e pragmalinguistico dei testi istroromanzi moderni (precipuamente rovignesi, pubblicati nell'antologia Istria Nobilissima) sono stati raccolti parecchi casi di ambiguità, cioè di frasi che ammettono due, tre o persino più di tre letture o interpretazioni. Mentre in certi nostri studi precedenti (Tekavčić 1982, 1987a, 1987b, 1987c) questi esempi sono stati per lo più soltanto citati o accompagnati tutt' al più da brevi commenti principalmente linguistici, nelle pagine che seguono intendiamo riprendere gli esempi concentrando la nostra attenzione da un lato sui fattori che generano l'ambiguità, dall'altro sui mezzi di disambiguazione. È appena necessario ricordare che l'ambiguità di una frase può esistere soltanto a livello puramente descrittivo, fuori contesto e/o situazione (per così dire, in vitro), mentre sparisce non appena la frase si inserisce nel contesto e/o nella situazione, diventando cosi un enunciato. Ciò vale naturalmente soprattutto per la lingua parlata ma anche, seppure in minore misura, per i testi. Perciò nel presente contributo aggiungiamo ad ogni esempio un breve quadro della situazione, sicché il lato pragmalinguistico e textlinguistico viene a trovarsi al primo piano. Il riassunto cerca di sistemare i tipi di frasi, i fattori di ambiguità $\mathrm{e}$ i mezzi per disambiguare.

\section{Analisi degli esempi'}

1) i nun siemo culpa nui, sa la dafoûnta la uò vusioû cuseî. (VI, 67) 'non abbiamo colpa noi se la defunta ha voluto così.'

Situazione: la Sora Batalita, un personaggio originale della vecchia Rovigno di tanti anni fa, ha formulato nel suo testamento il desiderio che per lei, invece della solita cerimonia funebre, si facesse una veglia allegra e un funerale con canti e balli. Le due sorelle, eredi di Batalita, si trovano in una situazione quanto mai imbarazzante: se ubbidiscono all'ultima volontà della defunta, a Rovigno ci sarà uno scandalo inaudito; se non ubbidiscono, perdono l'eredità. Una delle due sorelle pensa con paura alla reazione dei Rovignesi, l'altra risponde allora la frase citata.

Come in tutti gli esempi, è la situazione che ci permette di stabilire la lettura esatta. Dal punto di vista unicamente formale, cioè, l'esempio num. 1 contiene un periodo ipotetico sicché a prima vista l'esempio si potrebbe parafrasare così: 'Non

1 Gli esempi rispettano la grafia e le nostre correzioni si trovano fra parentesi quadre. Le cifre romane indicano il volume di Istria Nobilissima, quelle arabe la pagina. 
abbiamo colpa noi se Batalita ha voluto così' ossia, con l'inversione dell'asseveramento ${ }^{2}$ (o modalità): 'Abbiamo colpa noi se Batalita non ha voluto cosi' . Condensato in formule: non $A$ se $B=A$ se non $B$. In altri termini, la realizzazione dell'apodosi dipende dalla non-realizzazione della protasi e viceversa. Fin qui la descrizione puramente linguistica. La situazione ci mostra tuttavia che la prima lettura è insostenibile: l'ultima volontà di Batalita è un fatto, non soggetto più ad alcuna eventualità. Di conseguenza, la frase formalmente ipotetica in realtà è una frase dichiarativa (oggettiva o soggettiva a seconda della diatesi del verbo della matrice). La lettura esatta sarebbe dunque: 'Non abbiamo colpa noi del fatto che la defunta abbia voluto così'.

2) Tiremo a burdo la vila sa no i s'inpinemo. (VII, 222) 'Tiriamo a bordo la vela, se no ci riempiamo' [sc. di acqua]

Situazione: due fratelli, usciti a pescare, si trovano in pericolo di vita durante un forte temporale.

Anche qui abbiamo un periodo formalmente ipotetico, che in realtà è di un altro tipo e anche qui l'interpretazione ipotetica è esclusa dalla situazione, perché l'azione espressa nella apodosi non dipende da quella della protasi. La situazione si oppone a parafrasare l'esempio in forma di un periodo ipotetico effettivo ('Tiriamo a bordo la vela se non ci riempiamo di acqua' = 'Non tiriamo a bordo la vela se ci riempiamo di acqua'). Le parole sa no non sono scomponibili in $s a$, congiunzione ipotetica ('se') e no, negazione congiunta ('non'), ma costituiscono il sostituente profrastico corrispondente all'italiano se no (o anche sennò), che riassume in sé un'intera frase ipotetica ed equivale a 'altrimenti', 'in caso contrario', 'se non si esegue ciò che si dice nella frase precedente'. ${ }^{3}$ Interpretato così, l'esempio num. 2 è perfettamente logico: 'Tiriamo a bordo la vela, se no ( = altrimenti, se non lo facciamo) ci riempiamo di acqua'.

Un importante fattore di ambiguità è in quest'esempio l'omofonia di 'se non' e 'se no' in rovignese: ambedue le sequenze suonano sa no (var.: sa nuò), perché non c'è differenza formale sicura tra la negazione congiunta e la negazione autonoma (sostituente di frase). Un fattore di ambiguità secondario è.l'assenza della virgola, che trascrive la pausa.

3) Capeîso, par teîo zì el suoldo caval... (X,142) 'Capisco, per te è il soldo che vale...'

Situazione: una donna critica un'altra, che vuole dare la figlia in matrimonio ad un vecchio ricco, mentre le giovane è innamorata di un altro.

2 Per questo termine v. Weinrich 1966, specialm. pp. 11-12.

3 Per il concetto e il termine v. Gruppo di Padova 1979, pp. 339-340. 
La parola caval è un evidente errore di stampa (come ce ne sono tantissimi nei nostri testi ${ }^{4}$ ) per $c a v a l$ o anche $c^{\prime} a v a l^{5}$ 'che vale'. L'esempio ammette due letture, entrambe ugualmente accettabili fuori situazione:

a) 'per te è il soldo [ = il denaro] quello che vale'; l'enfasi è su 'soldo' e la frase è una frase scissa (si mette in risalto che per questa donna ciò che conta nella vita è il denaro e niente altro);

b) 'per te è [questo] il soldo che vale'; l'enfasi è su 'vale' oppure su 'questo' (se viene aggiunto) e la frase è una relativa restrittiva (si dice che per questa donna quello che vale è quel tale soldo e non un altro) ${ }^{6}$

L'ambiguità è dovuta qui alla struttura stessa della frase, e soltanto la situazione può imporre l'interpretazione esatta, che è beninteso la prima. Cfr. in seguito l'esempio num. 6.

4) veîn quà ch'i ta dazboûdo oûn guoto da veîn. $(\mathrm{X}, 152)$ 'vieni qui che/ché ti verso/versi [sc. io] un bicchiere di vino.'

Situazione: un uomo si è arrabbiato e ha litigato con una donna; un'altra donna cerca di calmarlo offrendogli un po' di vino.

Nel nostro quarto esempio le letture possibili sono tre, dovute a due fatti di ordine diverso: l'ambiguità della forma verbale dazboûdo e l'ambiguità della frase incastrata introdotta da $c h^{\prime}(=c a)$. Il rovignese neutralizza l'opposizione modale (indicativo $\sim$ congiuntivo) nella 1 persona del presente, essendo - $o$ la desinenza per ambedue i modi; di conseguenza, dazboûdo (di dazbudà, veneto desvodar 'vuotare' cioè 'versare da bere, mescere') vale tanto 'verso' quanto '(io) versi'. Quanto alla frase, la sua interpretazione dipende in parte da quella della forma dazboûdo: se questa viene intesa come congiuntivo, la frase incastrata è finale; se dazboûdo è indicativo, la frase introdotta da $c a$ può essere letta come causale performativa ${ }^{7}$ (che

4 Si veda Tekavčić 1983 , specialm. pp. $142-144, \S \S 13.2-13.7$.

5 Poiché in istroromanzo il clitico $a$ (soggetto dei verbi unipersonali o annunciatore del soggetto invertito) può apparire anche dopo 'che' (relativo o congiunzione), la sequenza ca nei testi va staccata in c'a in diversi casi (cfr. Tekavčić 1983, \$13.5).

- Per completare menzioniamo anche una terza lettura possibile, in cui 'per te' non sarebbe complemento di opinione o punto di vista ('per te' = 'dal tuo punto di vista' ecc.) ma complemento di termine ('per te' = 'destinato a te'). Quest'interpretazione è esclusa dalla situazione.

7 Adoperiamo il termine performativo nell'accezione che ha nella semantica generativa contemporanea in Italia, ad es.: «il performativo ci dice quale tipo di atto linguistico il parlante ha voluto compiere usando una certa frase» (Parisi - Antinucci 1977, p. 154). Secondo i due autori il performativo è «la configurazione semantica che specifica il tipo di intenzione comunicativa del parlante» (op. cit., p. 272). All'interno del performativo intendiamo la frase performativa nel senso di quanto si legge in Conte 1972, p. 164: «La frase performativa è una frase astratta sovrastante che non ha una realizzazione lessicale nella struttura superficiale, ma che determina gran parte della struttura superficiale delle frasi concrete d'una lingua». Le frasi dipendenti performative sono per noi quelle frasi che sono incastrate non nella matrice superficiale ma in quella sottostante, performativa. Cfr. per ciò Tekavčić 1987a. 
giustifica l'imperativo) oppure come un esempio di quella che altrove abbiamo denominato frase pseudosubordinata (Tekavčić 1987a, § 2.5): una frase cioè formalmente subordinata (incastrata), in realtà coordinata alla matrice ed esprimente una successione cronologica o tutt'al più una concatenazione logica. Le tre interpretazioni si lasciano parafrasare come segue:

a) 'Vieni qui affinché io ti versi un bicchiere di vino.'

b) 'Vieni qui ché [ = perché] ti verso un bicchiere di vino.'

c) 'Vieni qui, e ti verso un bicchiere di vino.'

L'esempio è molto simile a quello citato al num. 32 in Tekavčić 1987a (ven quà ca ti bivariè oun bicier [...] da quil bon, VII, 189). Comunque, nel nostro esempio num. 4 le tre letture sono ugualmente accettabili, e nemmeno la situazione ci aiuta. Soltanto la frequenza relativemente alta delle frasi pseudosubordinate nel corpus rovignese può parlare in favore della terza interpretazione.

5) i nûn zarì nuò l'infierno si [= s'i] fi la stufita $[=$ strufita]. $(\mathrm{X}, 157)$ 'non andrete no all'inferno se fate la strofetta. ${ }^{8}$

Situazione: ad una festa assiste fra altri convitati anche un prete; tutti cantano a turno, soltanto il prete si scusa dicendo che l'abito non gli permette di cantare, dopodiché una donna gli dice la frase citata.

È il terzo caso di periodo ipotetico nella nostra scelta di esempi, ma questa volta il periodo non è ipotetico solo formalmente: il primo dei due fatti (andare all'inferno) è effettivamente condizionato dal secondo (cantare una strofetta). Che ci siano ciò nonostante due letture è dovuto alla diversa incidenza (scope) della negazione.

a) Se la negazione nega l'intero periodo, cioè la concatenazione dei due fatti, si ha la prima interpretazione, parafrasabile così: 'non è vero che per aver cantato una strofetta andrete all'inferno'. Oppure così:

(non (andare all'inferno se cantare una strofetta)).

b) Se la negazione viene intesa come limitata alla sola apodosi (il fatto condizionato), la concatenazione dei due fatti rimane valida e si ha l'asseveramento complementare come negli esempi num. 1 e 2 . La parafrasi sarebbe 'non andrete all'inferno se cantate la strofetta' = 'andrete all'inferno se non cantate la strofetta'. Cioè:

(non andare all'inferno) (se cantare la strofetta).

Sebbene lo scopo principale, quello di indurre il prete a cantare, sia raggiunto in entrambi i casi, è owvio che la prima lettura è quella da preferirsi. Il periodo ipotetico equivale in sostanza ad un periodo causale.

8 L'omissione della preposizione $a$ è frequente nei testi rovignesi di Giusto Curto (il più noto degli scrittori rovignesi odierni) ed è una caratteristica della sua lingua. 
6) Siur'Anzula [...] a zì el fante ca la vol. (XII, 244) 'Signora Angela [...] è/c'è il fante che la vuole.'

Situazione: qualcuno avverte la signora Angela che il fattorino della posta batte alla porta e vuole parlarle.

In quest'ultimo esempio della prima parte della nostra scelta le interpretazioni che possiamo stabilire sono in numero assai elevato, non meno di cinque. Esse dipendono dal significato della forma verbale $a z i$ e dalla lettura della frase introdotta da $c a$.

a) Se la forma verbale $a z i$ equivale a 'è' e la frase è una relativa restrittiva, si ha la parafrasi 'è [sc. colui che batte alla porta] il fante che la vuole [e non un altro]'.

b) Se con lo stesso significato di $a z i$, la relativa viene letta come esplicativa, si ha quest'altra parafrasi: '[chi batte alla porta] è il fante, e questo fante la vuole'.

c) Sempre dando a $a z i$ il valore di 'è', la frase seguente ammette anche di essere interpretata come frase scissa, dunque con l'enfasi su 'fante', e la parafrasi è: 'è il fante che la vuole, e non qualcun altro'.

d) In istroromanzo a zi può corrispondere non solo a 'è' ma anche a 'c'è' (verbo cosiddetto esistenziale 'esserci'). ${ }^{9}$ Con questo significato è naturalmente esclusa una frase scissa, sicché restano le altre due letture. La prima corrisponde alla lettura a) (frase relativa restrittiva): 'c'è il fante che la vuole (e non un altro fante).'.

e) La quinta ed ultima lettura prospettata corrisponde alla lettura b) (frase relativa esplicativa): 'c'è il fante, e questo fante la vuole'.

In base a tutto il contesto, ci pare esclusa l'interpretazione c) (frase scissa) e le due letture della relativa come restrittiva. Rimangono dunque le interpretazioni b) $\mathrm{e}$ e), di cui ci decidiamo per la lettura e). La ragione è questa: non si tratta di risposta ad un'eventuale domanda di Angela 'Chi è', 'Chi batte?' ecc. (infatti, Angela non sente neppure che si batte alla porta), nel qual caso $a z i$ significherebbe 'è', ma dell'avvertimento dell'arrivo del fante, il che postula il significato esistenziale 'c'è'.

3. Ai sei esempi analizzati in modo alquanto dettagliato aggiungiamo una rapida occhiata su alcuni altri, per completare il quadro.

Nel nostro studio delle frasi dipendenti performative (Tekavčić 1987a) abbiamo citato al num. 39 l'esempio:

9 In seguito all'ineccepibile omofonia della 3 e 6 persona di tutti i paradigmi sia in istroromanzo che in veneto istriano, la sequenza $a z i$ vale anche 'sono' risp. 'ci sono', ma questo è ovviamente irrilevante in questa sede. 
7) Biteîna [...] la deî da spatà ca la xi ancura murieda. (III, 206) 'Bettina dice di aspettare che/perché è ancora giovane.'

Tralasciamo in questa sede l'analisi della seconda incastrata (per cui rimandiamo al citato studio) per constatare che la prima incastrata (cosiddetta implicita) ammette due letture, a seconda del duplice significato del verbo 'dire': 1 . enunciazione di un fatto (frase oggettiva dichiarativa), 2 . ingiunzione di un'azione (frase oggettiva volitiva). La situazione non ci è qui di nessun aiuto: qualunque delle due interpretazioni venga adottata, resta il fatto principale, la tergiversazione di Bettina, che si scusa di «non avere l'età»e in realtà non vuole sposare l'uomo che le cerca di imporre sua madre perché è innamorata di un altro.

Di conseguenza, la frase implicita si può leggere sia 'Bettina dice che (essa) aspetta' che 'Bettina dice che si aspetti'.

In un altro recente lavoro (Tekavčić 1987b) abbiamo citato (al num. 3, pag. 348) l'esempio:

8) Ch'i [ = Chi] ti vuoi ca la vago gratà mariuoli e bazase par doûta la veîta. $(X, 142)$ 'Che vuoi che essa vada a grattare camiciotti e bisacce per tutta la vita.'

Nel testo quest'esempio precede immediatamente il nostro esempio num. 3 (v. sopra). Sono le parole, piene di disprezzo per la vita dei contadini, dette da una madre che non vorrebbe dare la figlia in matrimonio ad uno di essi. Tutto il contesto assicura senz'alcuna esitazione la lettura di bazase come 'bisacce', ma fuori contesto la parola bazase corrisponde (in seguito a certe convergenze fonetiche a cui è dedicato appunto lo studio citato) altrettanto bene a 'baciarsi' (veneto basarse) e nemmeno la sintassi vi si opporrebbe, visto che 'baciarsi' sarebbe un infinito, coordinato a 'grattare' e dipendente dal verbo reggente 'andare a'. Soltanto il fattore extralinguistico (la situazione), assieme all'incompatibilità semantica, assicura la lettura soddisfacente.

Per non trascurare il secondo maggiore dialetto istroromanzo, il dignanese, e per illustrare nel contempo un tipo di ambiguità completamente diverso dai precedenti, anzi, contrario ad essi (e si vedrà subito perché), citiamo il seguente breve dialogo desunto dai testi dignanesi moderni aggiunti dal curatore Miho Debeljuh al Vocabolario di G. A. Dalla Zonca curato da lui (Dalla Zonca 1978). A p. 357 si legge:

9) A te pyås la pulęnta kul azì? - No ke no la me pyås! — Donka, la te pyås ku no la zi! 'Ti piace la polenta con l'aceto? - No, non mi piace! - Allora ti piace quando non c'è!'

Si tratta diun vero e proprio gioco di parole, basato sull'omofonia, in dignanese, di kul azì 'con l'aceto' (veneto co l'aseo) e ku la zi 'quando (essa) c'è' (veneto co la xe): 
ambedue le sequenze suonano / kulazi/. L'esempio si distingue da tutti i precedenti perché l'ambiguità sparisce non appena il testo si trascrive nella grafia convenziona$\mathrm{le}^{10}$ mentre sussiste nella forma pronunciata (negli esempi precedenti, al contrario, l'ambiguità era limitata alla forma scritta, fuori contesto e/o situazione, e disambiguata se pronunciata nella situazione adatta).

\section{Riassunti, confronti, conclusioni}

4.1 Per quanto riguarda la struttura sintattica della frase (negli esempi dove questa è rilevante), il tipo più rappresentato è quello ipotetico (esempi num. 1, $2 \mathrm{e}$ 5), seguito dalle frasi relative (esempi num. 3 e 6) e da quelle incastrate comuni (esempi num. 4 e 7). Negli esempi num. 8 e 9 il tipo di struttura sintattica è irrilevante.

4.2 La causa dell'ambiguità è la diversità delle interpretazioni sintattiche possibili, in tutti gli esempi meno l'esempio num. 8 (che è a livello morfologico) e l'esempio num. 9 (il quale è di ordine fonetico).

4.3 In certi casi ci sono fonti di ambiguità sussidiarie: l'omofonia di 'se no'e 'se non' nell'es. num. 2, la neutralizzazione dell'opposizione modale (nella 1 persona del presente) nell'es. num. 4, l'omofonia di 'è' e 'c'è' nell'es. num. 6.

4.4 Nella grande maggioranza dei casi la disambiguazione è assicurata dalla pragmatica (situazione) e dalla linguistica testuale (contesto). Là dove questi mezzi non riescono a disambiguare, nemmeno i mezzi linguistici stricto sensu sono di alcun aiuto. La superiorità dei fattori pragmalinguistici e textlinguistici nel funzionamento del linguaggio è dunque ovvia.

\section{OPERE CITATE}

Conte 1972: M. E. Conte, Vocativo ed imperativo secondo il modello performativo, in: Scritti e ricerche di grammatica italiana, Trieste, pp. 159-179.

Dalla Zonca 1978: G. A. Dalla Zonca, Vocabolario dignanese - italiano, a cura di Miho Debeljuh, Trieste.

Gruppo di Padova 1979: Gruppo di Padova: Aspetti dell'espressione della causalità in italiano, in: Società di Linguistica Italiana SLI 13/II: La grammatica, aspetti teorici e didattici, Roma, pp. 325-365.

Hall 1971: R. A. Hall jr., La struttura dell'italiano, Roma.

Istria Nobilissima: Antologia delle opere premiate, Primo Concorso d'arte e di cultura Istria Nobilissima (vol. I) 1968 - Diciottesimo Concorso ecc. (vol. XVIII) 1985, Trieste.

10 L'ambiguità rimarrebbe al contrario anche nella trascrizione, qualora si adottasse il sistema di trascrizione senza la divisione convenzionale del testo in parole, come ad es. in Hall 1971. 
Parisi - Antinucci 1977: D. Parisi - F. Antinucci, Elementi di grammatica, Torino. Tekavčić 1982: P. Tekavčić, Indirizzi linguistici attuali nel dominio istroromanzo, «Linguistica» 22, pp. 91-125.

Tekavčić 1983: P. Tekavčić, Problemi di grafia e di trascrizione nei testi istroroman$z i$, «Radovi Pedagoškog fakulteta u Rijeci OOUR nastavne djelatnosti Pula», num. 4 , pp. $135-149$.

Tekavčić 1987a: P. Tekavčić, Le frasi dipendenti performative nell'istroromanzo odierno, in: Romania et Slavia Adriatica, Festschrift für Žarko Muljačić, hrsg. von Günter Holtus and Johannes Kramer, Hamburg, pp. 373-388.

Tekavčić 1987b: $\mathrm{P}$. Tekavčić, Le convergenze e le divergenze fonetiche nell'istroromanzo (soprattutto rovignese) ed i loro riflessi nei testi, «Revue de Linguistique Romane» 51 , pp. 331-350.

Tekavčić 1987c: P. Tekavčić, Le frasi dipendenti nel rovignese attuale, «Travaux de Linguistique et de Littérature», 25/1, pp. 149-179.

Weinrich 1966: H. Weinrich, Per una linguistica della menzogna, «Lingua e stile» 1 , pp. 7-22.

Sažetak

SINTAKSA, SEMANTIKA, PRAGMATIKA U ISTROROMANSKIM TEKSTOVIMA: O NEKIM ZANIMLJIVIM SLUČAJEVIMA VIŠEZNAČNOSTI

U prilogu se, u okviru projekta studija suvremenih istroromanskih (prvenstveno rovinjskih) tekstova, proučavaju primjeri dvo- ili viక̌eznačnosti rečenica. Uz primjere uvijek se daje i potrebni opis situacije, jer situacija i/ili kontekst redovito omogućuju pravilno razumijevanje a time i razrješavaju višeznačnost (pa višeznačnosti redovito $i$ nema kad rečenica biva »uronjena« u kontekst $i$ /ili situaciju postajući tako iskaz). Većina analiziranih primjera dopušta dvije ili tri interpretacije (»čitanja《), neki i više (čak do pet). Po vrstama rečenica najčešće su pogodbene, zatim odnosne pa neki drugi tipovi. Višeznačnost je većinom na sintaktičkoj, samo u nekim malobrojnim primjerima na morfološkoj ili fonetskoj razini. Razrješenje višeznačnosti moguće je u velikoj većini primjerâ, zahvaljujući pragmatičkim i/ili tekstovnim faktorima; kada to nije moguće, specifično lingvistička sredstva ne mogu pomoći, što dokazuje superiornost pragmalingvističkih $\mathrm{i}$ tekstovnih faktora u funkcioniranju jezika. 